\title{
The synthetic-analytic character of color vision in the pigeon ${ }^{1}$
}

JOZEF COHEN

UNIVERSITY OF ILLINOIS

The pigeon's visual generalization gradient, as originally measured by Guttman and Kalish, suggests that the pigeon has an analyzing visual mechanism, unlike man's synthesizing visual mechanism but similar to his analyzing auditory mechanism. An experiment using a compound discriminative stimulus shows the pigeon's visual apparatus to be both synthesizing and analyzing.

The classical visual generalization gradient to wavelength by the pigeon, first recorded by Guttman \& Kalish (1956), is an enigma. The gradient was obtained by training pigeons to peck at a nearmonochromatic light of $550 \mathrm{~m} \mu$, the discriminative stimulus. The birds were shifted to a variable interval (VI) reinforcement schedule and they pecked steadily at about 100 responses per minute. The pigeons' "reflex reserve" was then sampled systematically by transilluminating the key with each of 12 test stimuli-near-monochromatic lights which spanned a large portion of the visible spectrum. The largest number of responses occurred to the original discriminative stimulus, and progressively fewer to stimuli whose wavelengths were increasingly different from the discriminative stimulus.

The pigeons' visual generalization gradient to wavelengths, while exhibiting a peak at the original training stimulus, is unexpected and surprising, for pigeons can do, apparently, what men cannot. The gradient suggests strongly that pigeons are equipped with a visual frequency analyzer in contrast to man's visual frequency synthesizer. The pigeons seem to "know" that the $490 \mathrm{~m} \mu-550 \mathrm{~m} \mu$ distance is greater than the $530 \mathrm{~m} \mu-550 \mathrm{~m} \mu$ distance, for they peck more slowly to $490 \mathrm{~m} \mu$ than to $530 \mathrm{~m} \mu$; pigeons are sensitive to wavelength (or frequency), for they order unerringly the several test stimuli according to wavelength. Men, who correlate wavelength with subjective hue, cannot order the hues (nor, of course, the wavelengths); indeed, students of experimental psychology must be taught the special hue sequence by a mnemonic device. The pigeon's visual frequency analyzer seems similar to man's auditory frequency analyzer; as a human ranks tones with higher frequencies as those subjectively higher and those with lower frequencies as those subjectively lower, a pigeon systematizes lights of higher and lower frequencies. Of this paradox, Guttman (1956) has written "It is quite remarkable that the ordinal relations between physical wavelengths are manifested in the generalization curve. The animal seems to know nothing about colors; he seems to know a very great deal about the physics of light... What is found for light would not be as surprising as if it were the case for sound."

It is to be noted that the most salient characteristic of human color vision is its synthesis of stimuli and its total resistance to analysis; human color vision never detects the constituent of a compound stimulus (comprised of two or more monochromatic stimuli) and two metameric stimuli (subjectively equivalent but physically dissimilar) are forever metameres -in adaptation, additive color mixture, etc. In contrast, the most salient characteristic of pigeon color vision, if it is an analyzer as the generalization gradient intimates, would be the capability of resolving compound stimuli; this is analogous to the human capability of hearing the separate components of two simultaneous tones.

A strategy for determining the synthetic-analytic character of pigeon color vision is evident. The bird may be trained on a partial schedule to peck at a compound discriminative stimulus comprised of two lights of, say, wavelengths $567 \mathrm{~m} \mu$ and $640 \mathrm{~m} \mu$, respectively. To a human observer, light of $567 \mathrm{m \mu}$ appears yellow-green, light of $640 \mathrm{~m} \mu$ appears red, light of $567 / 640 \mathrm{~m} \mu$ (in suitable proportions) appears orange with a dominant wavelength of $594 \mathrm{~m} \mu$; the pigeon's corresponding sensations, if any, are unknown. The bird may then be tested, with suitable randomness of order, with near-monochromatic lights spanning the spectrum. The emerging generalization gradient should be one of three types: (1) possessing a single peak between $567 \mathrm{~m} \mu$ and $640 \mathrm{~m} \mu$ (the peak will not necessarily coincide with $594 \mathrm{m \mu}$ ) indicating a synthetic color mechanism; (2) possessing two peaks -one at $567 \mathrm{m \mu}$ and the other at $640 \mathrm{m \mu}$-indicating an analyzing color mechanism; or (3) possessing three peaks-one at $567 \mathrm{~m} \mu$, one between 567 and $640 \mathrm{~m} \mu$, and one at $640 \mathrm{~m} \mu$-indicating a color mechanism which simultaneously both synthesizes and analyzes. Method and Procedure

This experiment used 16 domestic male white Carneaux pigeons, maintained at $65-70 \%$ of their free feeding weights. The birds were without prior experimental histories.

The stimuli, both near-monochromatic and compound, were provided by two Bausch and Lomb high intensity gating monochromators, utilizing $10 \mathrm{~m} \mu$ entrance and exit slits, which projected near-monochromatic lights, via a massive beam splitting prism, onto a translucent key in the bird's chamber. For compound stimuli, both 


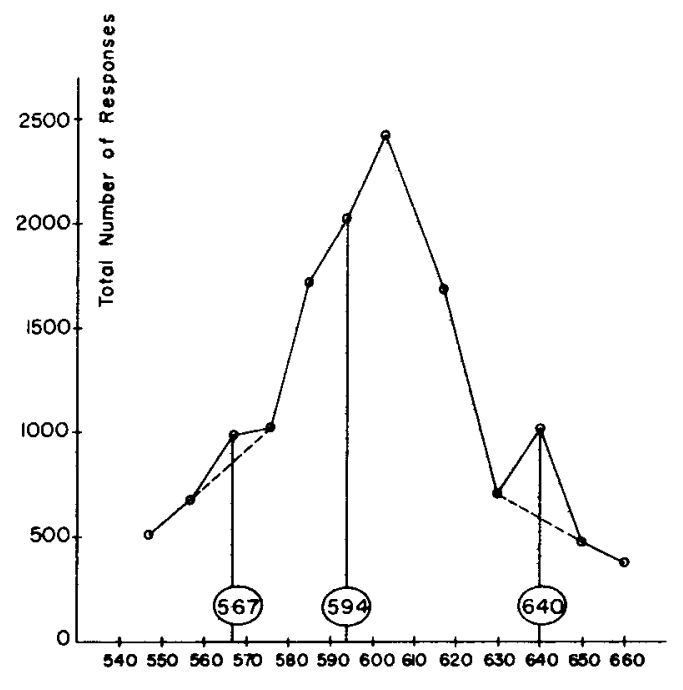

Wovelength in Millimicrons

Fig. 1. Total responses of 16 pigeons to 12 near-monochromatic test stimuli after training to a compound stimulus comprised of nearmonochromatic lights at $567 \mathrm{mu}$ and $640 \mathrm{mu}$.

monochromators were used; careful adjustment of the monochromators, prism, and key insured that the final compound-colored field, as seen by human observers, was uniform and without trace of the components. For near monochromatic stimuli, one monochromator was used.

The animal chamber was of standard size with a $3 / 4$ in. translucent key. The chamber was without illumination except that emerging from the key. The food hopper was illuminated, with a mirror, by the same monochromatic light or lights falling onto the key.

Each pigeon was first shaped, under room illumination, to peck at the key for a food reinforcement. A compound stimulus, comprised of near-monochromatic lights of $567 \mathrm{~m}_{\mu}$ and $640 \mathrm{~m} \mu$ was placed on the key, serving as the discriminative stimulus. The training perlod each day consisted of 151 min periods with the stimulus on, with a $20 \mathrm{sec}$ blackout between periods. The initial continuous schedule was gradually changed over 70 days to VR 60 , which was maintained for eight additional days. On the day following, each bird was given five warm-up periods with the discriminative stimulus. Then each bird was tested twice with near-monochromatic stimuli at 547, 557, $567,576,585,594,603,617,630,640,650$, and 660 $\mathrm{m} \mu$, in random order; there were, in total, 24 periods of 1 min with a $20 \mathrm{sec}$ blackout between them.

\section{Results and Discussion}

The combined responses of 16 pigeons to the 12 near-monochromatic test stimuli are shown in Fig. 1.

The generalization gradient is trimodal, indicating that the pigeon's color vision simultaneously both synthesizes and analyzes. The synthetic function is represented by the peak at $603 \mathrm{~m} \mu_{\text {, }}$ and is different from that of man, who equates this compound stimulus with a near-monochromatic stimulus of $594 \mathrm{~m} \mu$; human observers find the reddish-orange sensation of 603 $m_{\mu}$ to be markedly different than the orange sensation of $594 \mathrm{~m} \mu$. The analytic function is represented by the peaks at $567 \mathrm{~m} \mu$ and $640 \mathrm{m \mu}$, which are precisely at the locations of the components of the compound discriminative stimulus. Pigeons, then, apparently have the capability of resolving visual stimuli, as the original Guttman-Kalish generalization curve suggested.

The color vision of men and pigeons need not be the same, of course, especially since they evolved independently (Walls, 1942). The birds, it appears, emerged with the more sensitive apparatus in that their color vision yields more information. The avian integrating mechanism, as disclosed by the present experiment, differs modestly from the primate mechanism-enough so that the color mixture data and equations for primate vision cannot be applied. The avian analyzing mechanism differs totally from the primate mechanism, giving another dimension to the bird's visual apparatus.

\section{References}

GUTTMAN, N. The pigeon and the spectrum and other perplexities. Psychol Rep., 1956, 2, 449-460.

GUTTMAN, N., \& KALISH, H. I. Discriminability and stimulus generalization. J. exp. PsychoL, 1956, 51, 79-88.

WALLS, G. I. The Vertebrate Eye and its Adaptive Radiation. Bloomfield Hills, Michigan: Cranbrook Institute of Science, 1942. Note

1. This research was supported by the U. S. Department of Health, Education, and Welfare under grant number MH 08922-01-02. 